\title{
○emmestão
}

\section{Entrevista: Maria Cristiane Barbosa Galvão}

\author{
Marcos Vinícius Santos Carvalho Terra \\ Doutorando; Universidade Estadual Paulista, Marília, SP, Brasil. \\ marcosetica@gmail.com
}

\begin{abstract}
Resumo: Maria Cristiane Barbosa é professora do Departamento de Medicina Social da Faculdade de Medicina de Ribeirão Preto da Universidade de São Paulo. Doutora em Ciência da Informação pela Universidade de Brasília, realizou estágio na Universidade de Montreal. Além disso, foi pesquisadora associada da Universidade de Campinas e professora visitante do Departamento de Medicina de Família da Faculdade de Medicina da Universidade McGill e da Universidade de Málaga. Pesquisadora na área de informação em saúde, nesta entrevista, traz reflexões sobre a importância de pesquisadores e profissionais da informação na área da saúde no contexto da pandemia de Covid-19. A entrevista foi realizada em outubro de 2020.
\end{abstract}

Palavras-chave: Informação em saúde. Profissionais da informação. Pesquisadores. Covid-19.

\section{Introdução}

Maria Cristiane Barbosa é professora do Departamento de Medicina Social da Faculdade de Medicina de Ribeirão Preto da Universidade de São Paulo. Doutora em Ciência da Informação pela Universidade de Brasília, realizou estágio na Universidade de Montreal. Além disso, foi pesquisadora associada da Universidade de Campinas e professora visitante do Departamento de Medicina de Família da Faculdade de Medicina da Universidade McGill e da Universidade de Málaga. Pesquisadora na área de informação em saúde, nesta entrevista, traz reflexões sobre a importância de pesquisadores e profissionais da informação na área da saúde no contexto da pandemia de Covid-19. A entrevista foi realizada em outubro de 2020.

Como profissional e pesquisadora no campo da ciência da informação, como e quando surgiu seu interesse pelo campo da saúde?

Maria Cristiane Barbosa Galvão: Minha atuação no campo da saúde surgiu de uma necessidade do campo da saúde. Em 1992, no início da carreira, eu atuei como indexadora da base de dados Literatura Latino-Americana e do Caribe em 
Ciências da Saúde (LILACS). Este foi meu primeiro emprego. Um ano depois pedi minha exoneração desse cargo para me matricular no Curso de PósGraduação em Ciências da Comunicação na Escola de Comunicações e Artes da Universidade de São Paulo. Entre muitas idas e vindas pelo Brasil e exterior como docente em diferentes universidades, nos anos 2005, ministrei uma palestra vantagens e desvantagens do uso das classificações internacionais em saúde em um evento interdisciplinar e ao final desse evento recebi um convite de alguns docentes para atuar na Faculdade de Medicina de Ribeirão Preto da Universidade de São Paulo (FMRP-USP). Aceitei o convite e, como já era concursada nesta Universidade, solicitei minha transferência para esta unidade acadêmica. Tanto nos 90, quanto nos anos 2000, encarei essas oportunidades como um desafio profissional. Não foi algo previamente planejado e, portanto, demandou e demanda muito estudo, dedicação e aperfeiçoamento constante. É importante destacar que, fundada em 1951, a FMRP é uma unidade de referência no Brasil e exterior, congregando 16 departamentos acadêmicos, 7 cursos de graduação (Medicina, Ciências Biomédicas, Fisioterapia, Fonoaudiologia, Informática Biomédica, Nutrição e Metabolismo e Terapia Ocupacional), 24 programas de pós-graduação em diferentes especialidades da saúde. Gerencia vários hospitais e unidades de atenção secundária e primária em saúde onde cerca de 1 milhão de pacientes são atendidos e coopera para a formação de alunos de outras unidades acadêmicas como o Curso de Graduação em Biblioteconomia e Ciência da Informação. Minha atuação na FMRP perpassa a responsabilidade por várias disciplinas. Entre elas: Fundamentos em e-Saúde; Documentação em Saúde; Terminologias em Saúde; Fontes de Informação em Saúde; Comunicação e Difusão de Conhecimentos em Saúde, Tecnologias de Informação em Saúde. Por tudo isso, pode-se dizer que a FMRP é um cenário muito rico de possibilidades para o ensino, a pesquisa e a extensão de processos, serviços e produtos informacionais.

Qual a importância de um profissional ou um pesquisador da informação na área da saúde? Em um contexto pandêmico, quais contribuições os profissionais da informação podem oferecer à sociedade? 
Maria Cristiane Barbosa Galvão: O profissional da informação em saúde, em qualquer momento histórico, sempre está preparado e precisa estar preparado para atender demandas informacionais da população, de pacientes ${ }^{1}$, dos cuidadores de pacientes, dos profissionais da saúde ${ }^{2},{ }^{3}$, dos gestores da saúde ${ }^{4}$, como prefeitos, secretários de saúde, governadores, ministros e demais autoridades que atuam no contexto da saúde, bem como de pesquisadores, estudantes e aquelas demandas provenientes do complexo industrial da saúde $^{5}$, incluindo indústrias farmacêuticas, de equipamentos e serviços em saúde. Logo, o contexto da pandemia não alterou a atuação do profissional da informação que atua no campo da saúde, porém gerou um aumento significativo nas demandas informacionais. Assim, penso que as principais contribuições foram prover a informação necessária no momento correto e com a melhor qualidade possível.

De acordo com especialistas, $o$ isolamento social, durante a pandemia, pode trazer graves problemas emocionais para os indivíduos. Como os profissionais da informação podem contribuir para amenizar essa situação? Seria a biblioterapia uma alternativa?

Maria Cristiane Barbosa Galvão: A Organização Mundial da Saúde, em sua constituição firmada em $1948^{6}$, define saúde como não apenas a ausência de doenças ou enfermidades, mas inclui o bem-estar psicológico e social. Nesse sentido, não se pode entender que as demandas informacionais em saúde sejam apenas sobre enfermidades. Toda informação que possa melhorar o bem estar físico, mental e social das pessoas são bem vindas no campo da saúde. Nesse sentido, atividades como a contação de histórias ${ }^{7}$, a música ${ }^{8}$, o cinema, a dança e mesmo a realidade virtual e aumentada podem trazer sensações e emoções que promovam o bem-estar e a saúde das pessoas.

Um outro problema detectado durante a pandemia de Covid-19 foi a disseminação de notícias falsas. Qual o papel dos pesquisadores e dos profissionais da informação nesse cenário? 
Maria Cristiane Barbosa Galvão: Quando se atua no campo da saúde, é preciso uma formação informacional especializada para saber que tipo de informação em saúde possui um maior nível de evidência e que tipo de informação possui um baixo nível de evidência. Durante a pandemia, tanto a população, como alguns profissionais da informação e mesmo pesquisadores da informação não se atentaram aos critérios da qualidade informacional em saúde. Isso aconteceu também com os próprios profissionais da saúde. Essa disseminação de notícias falsas por tantas pessoas com diferentes tipos de formação é um alerta de que a competência informacional em saúde requer uma formação específica que pode ser alcançada por meio da oferta de cursos, disciplinas ${ }^{9}$, treinamentos especializados e educação continuada ${ }^{10}$.

\section{Durante a pandemia a importância do Sistema Único de Saúde (SUS) ficou} evidente. Como pesquisas em ciência da informação podem contribuir para melhorar o SUS?

Maria Cristiane Barbosa Galvão: O SUS ${ }^{11}$ é um sistema muito complexo que demanda todo tipo de ação dos profissionais da saúde. Tem um grande campo relacionado às informações clínicas registradas nos prontuários dos pacientes ${ }^{12} 13$ 1415 e, associada a essas informações, existe toda uma preocupação com a harmonização terminológica ${ }^{16} 17$ e a representação da informação de dados clínicos $^{18}$. Além da informação clínica, existe toda a necessidade da seleção, organização e disseminação de informações científicas ${ }^{19}$ para a tomada de decisão em saúde nos diferentes ambientes e contextos da saúde. E, como já foi mencionado, existe toda a preocupação e possibilidade de usar recursos informacionais e atividades culturais para garantir o bem estar físico, mental e social das pessoas ${ }^{20}$.

Quais são as barreiras e os facilitadores para atuação de profissionais da informação na área da saúde?

Maria Cristiane Barbosa Galvão: A principal barreira é o desconhecimento dos profissionais da informação sobre o campo da saúde. Os cursos de graduação e até mesmo alguns cursos de pós-graduação do campo da ciência da informação 
tendem a ser mais genéricos e não ofertam disciplinas obrigatórias ou optativas sobre o campo da saúde. Quando essa oferta acontece, nem sempre, o docente responsável teve oportunidade de obter uma formação na área da saúde. Muitas vezes desconhecem as profissões do campo da saúde, referindo em seus exemplos o médico enquanto uma entidade genérica e esquecem dos demais profissionais da saúde, suas funções e suas necessidades informacionais. Dessa forma, embora a formação clássica em informação seja de fundamental importância para os profissionais egressos desses cursos, ela não parece contemplar as especificidades requeridas pelo campo da saúde. Assim, os egressos do campo da ciência da informação precisam se desdobrar para conseguir ter acesso ao conhecimento necessário para atuar no campo da saúde por meio da realização de pós-graduação no campo da saúde, estágios em unidades da saúde e assim por diante. Para reverter esse quadro, é necessária uma compreensão da comunidade da ciência da informação de que os conceitos teóricos desenvolvidos pela área demandam prova de conceito para serem aplicados em contextos específicos e isso requer um conhecimento de campo mais aprofundado.

\section{Quais são os principais desafios para facilitar o acesso à informação na área da saúde?}

Maria Cristiane Barbosa Galvão: Para todos os falantes da língua portuguesa, um grande desafio é a barreira linguística. A informação em saúde é produzida, em sua maior parte, em língua inglesa. Nota-se que, embora existam vários tradutores automáticos, essas ferramentas são desconhecidas inclusive por muitos profissionais da saúde. Outro desafio é o custo da informação, pois grande parte da informação em saúde de melhor nível de evidência não possui acesso livre. Finalmente, o tempo é outro problema. Diariamente, são publicados centenas de artigos científicos no campo da saúde. Isso torna muito problemático selecionar e ter acesso às melhores informações. Somente sobre Covid-19, em meados de outubro de 2020, já foram indexados 63 mil artigos na Pubmed e 1,3 milhão de itens no Google Acadêmico. Esses números evidenciam o como é fácil ficarmos desatualizados no campo da saúde, mesmo se tivéssemos um dia com um número 
maior de horas.

Assim como os direitos à saúde e ao bem-estar, o direito à informação é um direito humano. Que relações podemos estabelecer entre esses direitos?

Maria Cristiane Barbosa Galvão: A conexão mais rápida de se estabelecer é com a dimensão ética da assistência em saúde que pressupõe que o paciente tenha poder e autonomia ${ }^{21}$ para tomar decisões sobre sua saúde. A autonomia do paciente só é possível se ele estiver suficientemente informado. Essa situação parece ilustrar como o direito à saúde se entrelaça com o direito à informação.

Que outros apontamentos acrescentaria sobre a importância de pesquisadores e profisssionais da informação na área da saúde, sobretudo, no contexto pandêmico?

Maria Cristiane Barbosa Galvão: De modo geral, neste momento, fazendo uma conexão com a questão anterior, uma dimensão importante é a dimensão ética. Se não há certeza que uma informação em saúde está correta é melhor não a divulgar. É preciso ter cautela, calma, uma sobriedade. Outra coisa importante é não ficar congestionando os canais de comunicação de profissionais da saúde, gestores e pacientes com informações redundantes sobre a pandemia. Não é porque surgiu um artigo novo sobre Covid-19 produzido por uma renomada instituição que essa informação precisa ser repassada para todos imediatamente. Aquela avaliação básica sobre o nível de evidência do novo artigo é fundamental. Nem todo artigo publicado tem qualidade ou é relevante ${ }^{22}$. É preciso se pensar também numa ética relacionada à produção do conhecimento. Vejo que alguns autores começaram a incluir o termo covid-19 em artigos apenas como um modo de ver seus trabalhos publicados ou de ter uma avaliação mais rápida e positiva de seus trabalhos. Em muitos casos, vê-se a superficialidade dessa produção e um certo oportunismo desnecessário e pouco saudável. Finalmente, minha expectativa é que toda essa vivência da pandemia de Covid-19 seja impulsionadora de novas propostas curriculares de graduação e pós-graduação para sairmos de um patamar nacional 
do "como o profissional da informação pode atuar no campo da saúde" para "atuações consolidadas do profissional da informação no campo da saúde" no Brasil, para além da atuação já consolidada em bibliotecas universitárias e acadêmicas da saúde. Por ser uma entrevista, deixei em notas alguns exemplos da minha atuação no campo da saúde. Espero que algumas dessas notas possam trazer algum tipo de inspiração para transformações no campo da ciência da informação ${ }^{23}$.

\title{
Interview: Maria Cristiane Barbosa Galvão
}

\begin{abstract}
Maria Cristiane Barbosa Galvão is Assistant Professor at the Department of Social Medicine at the Ribeirão Preto Medical School of the University of São Paulo. She has a PhD in Information Science by the University of Brasilia, with an internship at the University of Montreal. In addition, she was an associate researcher at the University of Campinas and a visiting professor at the Department of Family Medicine at the McGill University and at the University of Malaga. Researcher in the area of health information, in this interview, she brings reflections on the importance of researchers and information professionals in the health area in the context of the Covid-19 pandemic. The interview was conducted on October 2020.
\end{abstract}

Keywords: Health information. Health information researchers. Health information professionals. Covid-19.

\section{Declaração de autoria}

Concepção e elaboração do estudo: Não se aplica

Coleta de dados: Não se aplica.

Análise e interpretação de dados: Não se aplica.

Redação: (perguntas) Marcos Vinícius Santos de Carvalho Terra, (respostas)

Maria Cristiane Barbosa Galvão

Revisão crítica do manuscrito: Marcos Vinícius Santos de Carvalho Terra,

Maria Cristiane Barbosa Galvão

\section{Como citar}

GALVÃO, Maria Cristiane Barbosa. Entrevista: Maria Cristiane Barbosa

Galvão. [Entrevista concedida a] Marcos Vinícius Santos Carvalho Terra. Em

Questão, Porto Alegre, v. 27, n. 2, p. 440-448, abr./jun. 2021.

Doi: http://dx.doi.org/10.19132/1808-5245272.440-448 
1 Galvão, M.C.B.; Carmona, F.; Ricarte, I.L.M. Disseminando evidências em saúde em linguagem simples nas mídias sociais. In: Jornadas da Associação Portuguesa de Documentação e Informação de Saúde, 2018, Lisboa. Jornadas APDIS. Lisboa: APDIS, 2018. v. 2018. Disponível em: https://apdis.pt/publicacoes/index.php/jornadas/article/view/214

2 Galvão, M.C.B. et al. Disseminating health evidence summaries to increase evidence use in health care. Revista de Saúde Pública, v. 52, p. 1-10, 2018. DOI: http://dx.doi.org/10.11606/s1518- 8787.2018052000418.

3 Galvão, M.C.B. et al. O uso de evidências por enfermeiros brasileiros. Brazilian Journal of Information Science, v. 13, p. 5-13, 2019. Disponível em:

https://revistas.marilia.unesp.br/index.php/bjis/article/view/8873

4 Ricarte, I.L.M. et al. A implantação de iniciativas em saúde baseada em evidências: recomendações aos gestores da saúde. 2016. Disponível em:

https://www.researchgate.net/publication/292153017

5 Gadelha, Carlos Augusto Grabois. O complexo industrial da saúde e a necessidade de um enfoque dinâmico na economia da saúde. Ciênc. Saúde Coletiva, v. 8, n. 2, p. 521-535, 2003 DOI: http://dx.doi.org/10.1590/S1413-81232003000200015.

6 World Health Organization. Constitution. Geneva: WHO; 1948.

7 Galvão, M.C.B.; Carmona, F. A contação de história enquanto mecanismo de comunicação em saúde. 27 de outubro de 2016. In: Almeida Junior, O.F. Infohome [Internet]. Londrina: OFAJ, 2016. Disponível em: http://www.ofaj.com.br/colunas_conteudo.php?cod=1010

8 Galvão, M.C. B. et al. Disseminação de informação em saúde e música no contexto hospitalar pediátrico. Revista P2P e Inovação, v. 6, p. 212-223, 2019. DOI:

https://doi.org/10.21721/p2p.2019v6n1.p212-223

9 Galvão, M.C.B. Formação e prática médica: Os acervos virtuais como fonte de informação em saúde. In: Minha Biblioteca. Webinar Formação e Prática Médica, 2020. Disponível em: https://www.youtube.com/watch? $=$ =wpCYv6EYVlE\&t=2275s

10 Galvão, M.C.B. Métodos de pesquisa mistos e revisões de literatura mistas: uma janela de oportunidades científicas. Ribeirão Preto: Instituto de Estudos Avançados de Ribeirão Preto da Universidade de São Paulo, 2013. Disponível em: https://www.youtube.com/watch? $\mathrm{v}=\mathrm{Y} 3 \_\mathrm{i}$ 7FrsGKk

11 Galvão, M.C.B. Sistema Único de Saúde: aspectos que todo profissional da informação precisa conhecer. 29 de novembro de 2012. In: Almeida Junior, O.F. Infohome [Internet]. Londrina: OFAJ, 2012. Disponível em: http://www.ofaj.com.br/colunas_conteudo.php?cod=719

12 Galvão, M.C.B.; Ricarte, I.L.M. Prontuário do paciente. 1. ed. Rio de Janeiro: Guanabara Koogan, 2012. v. 1. 344p.

13 Galvão, M.C.B.; Ricarte, I.L.M. Alinhamentos necessários entre o registro eletrônico de saúde e o sistema de saúde. Informação \& Informação (Online), v. 22, p. 426-455, 2017. Disponível em: http://www.uel.br/revistas/uel/index.php/informacao/article/download/20190/22741

14 Galvão, M.C.B.; Ricarte, I.L.M. O prontuário eletrônico do paciente no século XXI: contribuições necessárias da ciência da informação. InCID: Revista de Ciência da Informação e

Documentação, v. 2, p. 77-100, 2011. DOI: https://doi.org/10.11606/issn.2178-2075.v2i2p77-100

15 Galvão, M.C.B.; Ricarte, I.L.M. O prontuário eletrônico do paciente em escala nacional: o caso australiano. InCID: Revista de Ciência da Informação e Documentação, [S. 1.], v. 10, n. 1, p. 244- 264, 2019. DOI: https://doi.org/10.11606/issn.2178-2075.v10i1p244-264.

16 Galvão, M.C.B. Terminologias e comunicação em saúde: uma questão de vida ou morte. Infohome, Londrina, 012014.2 Disponível em: http://www.ofaj.com.br/colunas_conteudo.php? cod= 834

17 Galvão, M.C.B. Uso de linguagens de especialidade na prática profissional: versão 2019. In: Márcia Cubas; Miriam Nóbrega. (Org.). Classificação Internacional para a Prática de Enfermagem CIPE®: versão 2019. Porto Alegre: Artmed, 2019

18 Galvão, M.C.B. A leitura documentária e a indexação de documentos da área de saúde. In: Fujita, Mariângela Spotti Lopes et al. (Org.). Modelos de leitura documentária para indexação: 
abordagens teóricas interdisciplinares e aplicações em diferentes tipos de documentos. Marília: UNESP, 2019. DOI: https://doi.org/10.36311/2020.978-65-86546-07-1.p173-194

19 Galvão, M.C.B.; Ricarte, I.L.M. Revisão sistemática da literatura: conceituação, produção e publicação. Logeion Filosofia da Informação, v. 6, p. 57-73, 2019. DOI:

https://doi.org/10.21728/logeion.2019v6n1.p57-73

20 Terra, M.V.S. de C.; Mamede, J. R.; Galvão, M.C.B. Relacionamentos entre a mediação da informação e o processo de humanização da assistência em saúde: um começo de conversa. Infohome, Londrina, 29 set. 2016.2 Disponível em: https://www.ofaj.com.br/colunas_conteudo.php? cod=1005

21Galvão, M.C.B. O prontuário do paciente enquanto suporte para a autonomia e o empoderamento do paciente. Infohome, Londrina, 03 out. 2013. Disponível em: https://www.ofaj.com.br/colunas_conteudo.php?cod=782

22 Galvão, M.C.B. et al. The clinical relevance of information index (CRII): assessing the relevance of health information to the clinical practice. Health Information and Libraries Journal, v. 30, p. 110-120, 2013. DOI: https://doi.org/10.1111/hir.12021

23 Galvão, M.C. B Disciplinas sobre informação em saúde ministradas entre 2008-2018 na Universidade de São Paulo. Ribeirão Preto : USP, 2019. Disponível em:

http://eprints.rclis.org/38440/ 